A COMPARISON OF FUNCTIONAL AND

ECHOCARDIOGRAPHIC OUTCOMES IN NICE COMPLIANT AND NON-COMPLIANT PATIENTS UNDERGOING CRT IN THE REAL WORLD

doi:10.1136/heartjnl-2011-300198.94

${ }^{1} \mathrm{~S}$ J Russell, ${ }^{1} \mathrm{I}$ Rees, ${ }^{2} \mathrm{P}$ O'Callaghan, ${ }^{2} \mathrm{Z}$ R Yousef. ${ }^{1}$ Wales Heart Research Institute, Cardiff, UK; ${ }^{2}$ University Hospital of Wales, Cardiff, UK

Introduction The National Institute for Clinical Excellence (NICE) define a population of patients that are most likely to respond to cardiac resynchronisation therapy (CRT) and have a favourable health economic profile. Current NICE criteria (technology appraisal; TA120) for CRT include: NYHA class III or IV symptoms despite optimal medical therapy, sinus rhythm, ejection fraction $\leq 35 \%$, and either ORS duration $>150 \mathrm{mS}$ alone or $120-149 \mathrm{mS}$ together with echocardiographic (echo) evidence of mechanical dyssynchrony. Several randomised clinical trials however have consistently reported beneficial effects of CRT in patients outside current NICE guidelines. In our centre, potential CRT patients are discussed at a multi-disciplinary team (MDT) meeting attended by a heart failure specialist, electrophysiologist, interventional cardiologist, cardiac surgeon and hospital manager. CRT is offered where there is consensus agreement that the individual patient is likely to benefit. This individualised and evidence based approach provides for a comparison of outcomes in NICE compliant (NICE:+ve) and NICE: -ve patients (patients with a clinical need and evidence base supporting CRT, but who do not meet NICE criteria).

Methods Our unit operates an integrated CRT service with preassessment, implantation, and follow-up components. Pre-assessment includes clinical evaluation and baseline echo (EF: ejection fraction, and ESV: left ventricular end-systolic volume) and func-

Abstract 94 Table 1 Characteristics of NICE compliant and noncompliant patients

\begin{tabular}{lll}
\hline & NICE:+ve & NICE: - ve \\
\hline Age: years (SD) & $65(11)$ & $66(11)$ \\
Male: \% & 83 & 90 \\
Ejection fraction: \% (SD) & $22(7.1)$ & $24(7.2)$ \\
QRS duration: mS (SD) & $164(26)$ & $158(37)$ \\
CRT-Defibrillator: \% & 57 & 46 \\
\hline
\end{tabular}

Abstract 94 Table 2 Outcomes in NICE compliant and noncompliant patients

\begin{tabular}{lclll}
\hline & $\begin{array}{l}\text { NICE:+ve } \\
\text { (n=89) }\end{array}$ & $\begin{array}{l}\text { p value } \\
\text { (baseline v } \\
\mathbf{6} \text { months) }\end{array}$ & $\begin{array}{l}\text { NICE: - ve } \\
\text { (n=50) }\end{array}$ & $\begin{array}{l}\text { p value } \\
\text { (baseline v } \\
\mathbf{6} \text { months) }\end{array}$ \\
\hline OOL (score): baseline (SD) & $58.2(23.6)$ & & $63.5(31.7)$ & \\
OOL (score): 6 months (SD) & $40.1(25.0)$ & $<0.001$ & $38.9(25.6)$ & $<0.001$ \\
6MWT (m): baseline (SD) & $216.4(118.3)$ & & $208.5(131.8)$ & \\
6MWT (m): 6 months (SD) & $324.5(131.2)$ & $<0.001$ & $291.5(127.5)$ & $<0.01$ \\
V02 (ml/kg/min): baseline (SD) & $13.2(5.6)$ & & $12.3(4.0)$ & \\
V02 (ml/kg/min): 6 months (SD) & $14.4(3.3)$ & 0.24 & $12.8(4.2)$ & 0.64 \\
Ejection fraction- baseline (SD) & $21.5(7.1)$ & & $24.3(7.2)$ & \\
Ejection fraction- 6 months (SD) & $30.7(10.4)$ & $<0.001$ & $31.4(6.6)$ & $<0.001$ \\
End-systolic volume- & $185.1(79.3)$ & & $177.2(67.2)$ & \\
baseline /ml (SD) & & & & \\
End-systolic volume- & $144.6(73.5)$ & 0.02 & $135.2(57.2)$ & 0.01 \\
6 months /ml (SD) & & & & \\
\hline
\end{tabular}

tional characterisation: a) Minnesota quality of life score (QoL), b) 6 min walk test (6MWT), and c) peak oxygen consumption on cardiopulmonary exercise test (VO2). Follow-up at 3 and 6 months post CRT includes clinical evaluation, device/medical optimisation, and reassessment of echo and functional outcomes. This study involves a retrospective analysis of our CRT database and compares outcomes in NICE: +ve and NICE: -ve patients.

Results Between January 2007 and December 2009, 253 patients received CRT. Complete paired data comparing baseline and 6 month functional and echo data are available for 139 patients; 89 NICE:+ve and 50 NICE: -ve (Abstract 94 table 1). Exclusions for the NICE: -ve patients included: atrial fibrillation $(n=19)$, ORS 120-149 mS without mechanical dyssynchrony ( $n=12)$; ORS $<120 \mathrm{mS}(\mathrm{n}=5)$; pacemaker upgrades $(\mathrm{n}=9)$. An additional 5 patients with right bundle branch block and otherwise NICE CRT compliance are analysed as NICE: - ve in this study. Compared to baseline, 6-month outcomes were similar and significantly improved in both NICE:+ve and NICE: -ve groups (Abstract 94 table 2).

Conclusions We observed significantly favourable and similar functional and echocardiographic responses to CRT in patients meeting and not meeting current NICE criteria for CRT. Guidelines should guide therapy but ultimately each therapy should be individualised and evidence based.

\section{IMPAIRED CARDIAC ENERGETICS IN DILATED CARDIOMYOPATHY: MAGNETIC RESONANCE SPECTROSCOPY AT 3T}

doi:10.1136/heartjnl-2011-300198.95

${ }^{1} \mathrm{R}$ M Beadle, ${ }^{2} \mathrm{~L}$ K Williams, ${ }^{2} \mathrm{M}$ Kuehl, ${ }^{2} \mathrm{~S}$ Bowater, ${ }^{2} \mathrm{~K}$ Abozguia, ${ }^{2} \mathrm{~F}$ Leyva Leon, ${ }^{1} \mathrm{M} P$ Frenneaux. ${ }^{1}$ University of Aberdeen, Aberdeen, UK; ${ }^{2}$ University of Birmingham, Birmingham, UK

Introduction The aim was to measure the cardiac phosphocreatine to ATP (PCr/ATP) ratio non-invasively in patients with dilated cardiomyopathy and normal controls, and to correlate the patient's results to symptom status, ejection fraction (EF) and quality of life scores. Dilated cardiomyopathy is known to be associated with cardiac energy deficiency. Magnetic resonance spectroscopy (MRS) has been proposed as a non-invasive method of assessing cardiac energetics and as a method of measuring response to therapy. Interrogation at high field strength improves signal to noise ratio.

Methods 32 patients and 22 control subjects were studied using phosphorus-31 (31P) MRS and patients were classified to NYHA symptom class. In vivo energetics were measured using a commercially available Philips Achieva 3 Tesla scanner and dedicated 31P coil with ISIS volume selection. Java Magnetic Resonance User Interface (jMRUI) was used for analysis. Furthermore, all patients completed a Minnesota Living with Heart Failure (MLWHF) score and underwent echocardiography. LVEF was measured using biplane Simpson's method.

Results The PCr/ATP ratio was significantly reduced in patients (1.35 \pm 0.31$)$ compared with control subjects $(1.90 \pm 0.40 ; p<0.005)$. The PCr/ATP ratio was correlated with NHYA class $(r=-0.68$, $\mathrm{n}=32, \mathrm{p}<0.0005$ ). No correlation was found with LVEF or MLWHF score.

Conclusions This study confirms the presence of energy deficiency in dilated cardiomyopathy as measured by MRS at 3T. The energy status correlates strongly with symptom status but not with ejection fraction nor quality of life score. Cardiac energetic status is directly proportional to symptoms status and therefore any treatments targeted to improve cardiac energetics may improve patient symptoms in dilated cardiomyopathy. 

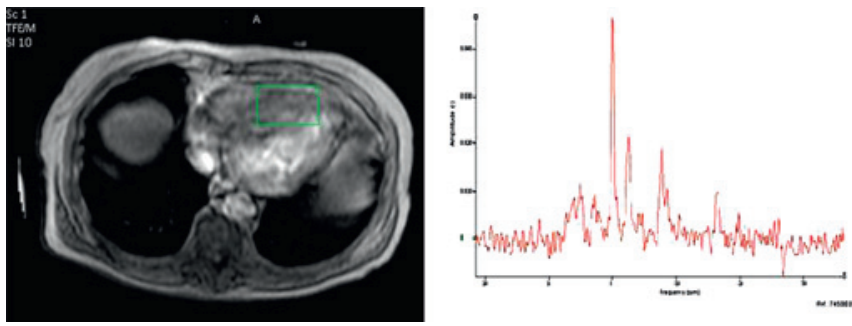

Abstract 95 Figure 1
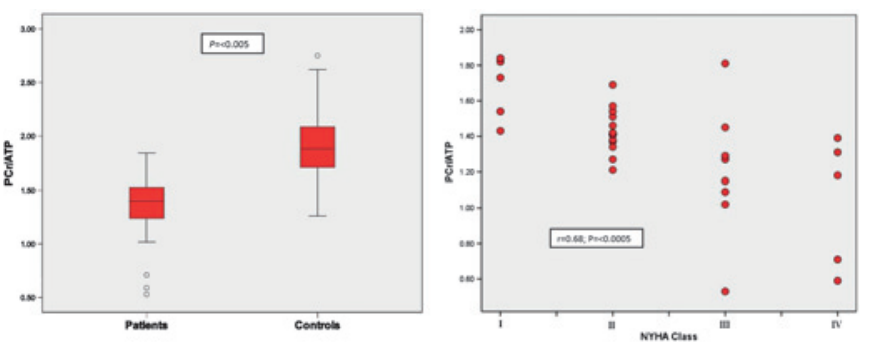

Abstract 95 Figure 2

\section{A TEST TO CONFIRM MAXIMAL OXYGEN UPTAKE IN CHRONIC HEART FAILURE PATIENTS WITHOUT THE NEED FOR SECONDARY CRITERIA}

doi:10.1136/heartjnl-2011-300198.96

T S Bowen, D T Cannon, G Begg, V Baliga, K K Witte, H B Rossiter. University of Leeds, Leeds, UK

Cardiopulmonary exercise testing for peak oxygen uptake $\left(\mathrm{VO}_{2 \text { peak }}\right)$ is widely used to evaluate severity, pathophysiology and prognosis in patients with chronic heart failure (CHF). $\mathrm{AVO}_{2 \text { peak }} \leq 14$ (or 12 with $\beta$-blocker) $\mathrm{ml} / \mathrm{kg} / \mathrm{min}$ is associated with increased mortality and is a key criterion for cardiac transplant listing. A symptom-limited exercise test, however, may elicit a $\mathrm{VO}_{2 \text { peak }}$ lower than the maximum physiological limit $\left(\mathrm{VO}_{2 \mathrm{max}}\right)$; the latter commonly "confirmed" using the secondary criterion of respiratory exchange ratio (RER) $>1.05$. RER, however, is sensitive to the test format. We, therefore, determined if a ramp-incremental (RI) step-exercise (SE) (or RISE) test could determine $\mathrm{VO}_{2 \max }$ in CHF patients without using RER, by satisfying the criterion that two different work rates are terminated at the same $\mathrm{VO}_{2 \text { peak. }}$. Twenty-one male CHF patients (NYHA class I: $\mathrm{n}=3$, II: $\mathrm{n}=16$, and III: $\mathrm{n}=1$ ) initially performed a modified Bruce treadmill test. Patients then completed a symptom-limited RISE95 cycle ergometer test in the format: RI (4-18 W/min; 10 min); 5 -min recovery $(10 \mathrm{~W})$; SE ( $95 \%$ of peak RI work rate). Thirteen of these patients also performed RISE95 tests using slow (RI 3-8 W/ $\mathrm{min}$; $\sim 15 \mathrm{~min}$ ) and fast (RI 10-30 W/min; $\sim 6 \mathrm{~min}$ ) ramp rates. $\mathrm{VO}_{2}$ and RER were measured breath-by-breath by a mass spectrometer and turbine (MSX, NSpire, UK). Peak $\mathrm{VO}_{2}$ and RER were compared within-subjects, between RI and SE, by unpaired t test of the final 12 breaths of exercise. This approach allowed $\mathrm{VO}_{2 \max }$ and its associated $95 \%$ confidence limits to be estimated. $\mathrm{VO}_{2 \text { peak }}$ was similar ( $p>0.05)$ in treadmill and cycle exercise (mean \pm SD: $16.2 \pm 2.7$ vs $15.0 \pm 3.2 \mathrm{ml} / \mathrm{kg} / \mathrm{min}, \mathrm{n}=20$, respectively), despite $R E R$ being greater in cycling $(1.08 \pm 0.12$ vs $1.15 \pm 0.09 ; p<0.05)$. As a group, $\mathrm{VO}_{2 \text { peak }}$ was similar $(\mathrm{p}>0.05)$ between $\mathrm{RI}$ and SE (mean \pm SD: $14.6 \pm 3.2$ vs $14.9 \pm 3.2 \mathrm{ml} / \mathrm{kg} / \mathrm{min}, \mathrm{n}=21$ ). A within-subject comparison, however, revealed that the $\mathrm{VO}_{2 \max }$ criterion was met in 14 of 21 patients (measurement sensitivity range $0.6-3.8 \mathrm{ml} / \mathrm{kg} / \mathrm{min}$ ), despite RER being $>1.05$ in the remaining 7 (1.16 \pm 0.09$)$. There was no effect of ramp rate on $\mathrm{VO}_{2 \text { peak }}(\mathrm{p}>0.05)$, however $\mathrm{RER}$ was greater $(p<0.05)$ in the fast ramp $(1.24 \pm 0.09)$ compared to the slow
(1.12 \pm 0.06$)$. The single-visit RISE95 test incorporating incrementaland step- exercise phases, each to the volitional limit, was well tolerated by CHF patients: The SE phase was contraindicated in only 3 of the 47 tests. The RISE95 detected $\mathrm{VO}_{2 \max }$ in 14 of 21 patients with a sensitivity of $\sim 10 \%$ (ie, similar to healthy subjects), and without the need for secondary criteria or incidence of false-positive. In contrast, the end-exercise RER was sensitive to both modality and ramp rate and provided a false-positive for $\mathrm{VO}_{2 \max }$ attainment in every incidence. Therefore, the RISE95 protocol provides a robust measure of $\mathrm{VO}_{2 \max }$ in CHF patients, to within an individuallydefined CI without dependence on secondary criteria.

\section{INCREASING SKELETAL MUSCLE OXYGENATION BY PRIOR MODERATE-INTENSITY EXERCISE INCREASES AEROBIC ENERGY PROVISION IN CHRONIC HEART FAILURE}

doi:10.1136/heartjnl-2011-300198.97

T S Bowen, D T Cannon, S R Murgatroyd, K K Witte, H B Rossiter. University of Leeds, Leeds, UK

Rapid adaptation of pulmonary oxygen uptake $\left(\mathrm{VO}_{2}\right)$ at exercise onset reduces the reliance on limited anaerobic energy stores and is associated with increased exercise tolerance. These $\mathrm{VO}_{2}$ kinetics, however, are slow in patients with chronic heart failure (CHF). This could be due to limitations in the control of muscle $\mathrm{O}_{2}$ consumption and/or $\mathrm{O}_{2}$ delivery. Recent evidence in CHF of a transient overshoot in microvascular deoxygenation at exercise onset supports the latter. As prior exercise is known to increase muscle blood flow in healthy individuals, we examined whether it could attenuate the fall in microvascular deoxygenation and speed $\mathrm{VO}_{2}$ kinetics on transition to moderate exercise in CHF patients. Thirteen CHF patients (NYHA class I: $n=3$, II: $n=9$, and III: $n=1$ ) performed a ramp test on a cycle ergometer for estimation of lactate threshold (LT) and $\mathrm{VO}_{2 \max }$. Patients subsequently repeated two 6-min moderate-intensity exercise transitions (bout 1, bout 2) from rest to $90 \% \mathrm{LT}$, separated by 6 -min of rest. Measurements included breath-by-breath $\mathrm{VO}_{2}$ using a turbine and mass spectrometer (MSX, NSpire, UK), and tissue oxygenation index (TOI) of the vastus lateralis by spatially resolved near-infrared spectroscopy (NIRO200, Hamamatsu, Japan). The exponential timeconstant $(\tau)$ for TOI and phase II VO2 were estimated using nonlinear least-squares regression. The $\tau \mathrm{VO}_{2} / \tau \mathrm{TOI}$, or "kinetic index", was taken to reflect the relative matching of muscle oxygenation to its instantaneous requirement. LT and $\mathrm{VO}_{2 \max }$ were $9.9 \pm 1.7$ (mean $\pm \mathrm{SD}$ ) and $15.0 \pm 3.2 \mathrm{ml} / \mathrm{kg} / \mathrm{min}$, respectively. Prior exercise increased resting TOI by $10 \pm 3 \%(p<0.05)$, attenuated the transient overshoot in muscle deoxygenation by $\sim 50 \%(p<0.05)$ and slowed the rate of deoxygenation in the transient ( $\tau$ TOI: $10 \pm 1$ vs $21 \pm 13 \mathrm{~s}$; $\mathrm{p}<0.05)$. Both $\tau \mathrm{VO}_{2}(46 \pm 20$ vs $39 \pm 18 \mathrm{~s} ; \mathrm{p}<0.05)$ and the kinetic index $(4.5 \pm 1.8$ vs $2.2 \pm 0.9 ; \mathrm{p}<0.05)$ were reduced following prior exercise. $\tau \mathrm{VO}_{2}$ was well correlated to the kinetic index $\left(\mathrm{R}^{2}=0.92\right)$ in bout 1 . However, although a lower $\tau \mathrm{VO}_{2}$ was typically reflected in a reduced kinetic index in bout $2, \mathrm{VO}_{2}$ kinetics remained slowed in 4 patients. These patients had a higher NYHA class $(2.3 \pm 0.5$ vs $1.6 \pm 0.5 ; \mathrm{p}=0.06)$ and greater initial $\tau \mathrm{VO}_{2}(62 \pm 17$ vs $33 \pm 9 \mathrm{~s} ; \mathrm{p}<0.05)$ than the others. In CHF prior moderate-intensity exercise improved the dynamic matching of muscle oxygenation to its instantaneous requirement and speeded $\mathrm{VO}_{2}$ kinetics in all patients. This suggests that slow $\mathrm{VO}_{2}$ kinetics in $\mathrm{CHF}$ are due, at least in part, to a dynamic limitation in $\mathrm{O}_{2}$ delivery. However, this approach revealed an apparent limitation in the control of muscle $\mathrm{O}_{2}$ consumption in the most severe patients, which was only partly ameliorated by improving $\mathrm{O}_{2}$ delivery. Nevertheless, these findings suggest that an acute intervention to improve muscle oxygenation can increase aerobic energy provision on transition to exercise in CHF patients. 